\title{
Neuropsychological correlates of cerebellar volumes in multiple sclerosis: an MRI volumetric analysis study
}

\author{
Paraskevi Iliadou ${ }^{1, *}$, Christos Bakirtzis ${ }^{2}$, Panagiotis loannidis ${ }^{2}$, Katherine Possin ${ }^{3,4}$, Stelios Zygouris ${ }^{5}$, Styliani-Aggeliki Sintila ${ }^{2}$, \\ Nikolaos Crigoriadis ${ }^{2}$, Eleni Aretouli ${ }^{6}$
}

${ }^{1}$ Lab of Cognitive Neuroscience, School of Psychology, Aristotle University of Thessaloniki, 54124 Thessaloniki, Greece

${ }^{2}$ The Multiple Sclerosis Center, 2nd Department of Neurology, AHEPA University Hospital, Aristotle University of Thessaloniki, 54124 Thessaloniki, Greece

${ }^{3}$ Department of Neurology, Memory and Aging Center, University of California, San Francisco School of Medicine, San Francisco, CA 94102, USA

${ }^{4}$ The Clobal Brain Health Institute, University of California, San Francisco, San Francisco, CA 94102, USA and Trinity College Dublin, 2 Dublin, Ireland

${ }^{5}$ Centre for Research and Technology Hellas/Information Technologies Institute, 57001 Thessaloniki, Greece

${ }^{6}$ School of Psychology, University of loannina, 45110 loannina, Greece

*Correspondence: evi.iliadou@yahoo.com (Paraskevi Iliadou)

\section{DOI:10.31083/j.jin2101013}

This is an open access article under the CC BY 4.0 license (https://creativecommons.org/licenses/by/4.0/).

Submitted: 28 April 2021 Revised: 5 July 2021 Accepted: 21 July 2021 Published: 28 January 2022

The hallmark of Multiple Sclerosis (MS) pathophysiology is the damage to the myelin sheath around axons. The cerebellum is a predilection site for demyelination with a well-recognized role in motor and a rather understudied contribution to cognitive functions. The aim of this study is to investigate patterns of cerebellar grey and white matter pathology, expressed as reduced volume, as well as cortical thickness and their potential contribution to cognitive performance and disability status of patients with MS. 24 patients with MS underwent extensive neuropsychological assessment using paper and pencil tests and the Brain Health Assessment (BHA) tablet-based battery. Cerebellar lobular volumes and thickness were calculated using a volumetric analysis with automated segmentation of the cerebellum and its lobules. The main findings are a reduction of cerebellar grey matter (CCMV) and white matter volumes (CWMV) in lobule $\mathrm{X}$ and a widespread cerebellar cortical thinning in patients. Overall disease severity and neurological disability, assessed with the Expanded Disability Status Severity Scale, was correlated with fatigue and information processing speed tasks, but not with CGMV and CWMV. CWMV and CGMV of lobule I-II was negatively correlated with information processing speed, as well as visuospatial memory tests and, finally, inverse cortical thinning associations were noted between the whole cerebellum, lobule I-II, lobule III, lobule VI, Crus I, lobule VIIIA and information processing speed and verbal fluency tasks. The inverse associations observed may represent a compensatory mechanism activated in MS engaging additional high-level cortical areas functionally interconnected with the damaged cerebellum, in order to cope with the cognitive demands of a task.

Keywords

Multiple sclerosis; Cognitive impairment; Cerebellum; Volumetry analysis

\section{Introduction}

Multiple Sclerosis (MS) is a heterogeneous, neurodegenerative disease, characterized by the presence of inflammation, demyelination and loss of axons and neurons in the Central Nervous System (CNS) that leads to progressive physi- cal disability and cognitive impairment. MS can cause a wide spectrum of signs and symptoms, depending on the location and the extent of the lesions in the CNS affecting the physical, emotional and cognitive functioning of persons with Multiple Sclerosis (PwMS). Cognitive impairment is estimated to affect $40 \%-65 \%$ of PwMS [1] and may be present even in the absence of physical disability. The cognitive domains affected in MS can range across patients, but typically include attention and concentration, executive functions, information processing speed, learning and memory and visuospatial abilities. Cognitive impairment increases individuals' disease burden and is directly related to social functioning, employability and independence [2].

Progressive and widespread brain volume loss is a common finding in MS. The cerebellum, in particular, is a site of atrophy in this population of patients [3], even at the early stages of the disease [4]. The mechanisms contributing to cerebellar atrophy in PwMS include demyelination, axonal/neuronal loss [5] and synaptic reduction [4]. Although demyelination affects more often the cerebellar white matter and the deep nuclei, histopathological studies have shown demyelination in the cortical cerebellar grey matter, as well, which could be even more extended, on average covering from $14 \%$ up to $90 \%$ of the total cerebellar GM atrophy for a review see Parmar et al. [6]. The few available MRI studies have demonstrated a significant reduction in cerebellar GM and WM volume in PwMS compared to healthy controls, which is related not only to impaired motor function, but also impaired performance on cognitive tests. Anatomical evidence suggests a dichotomy of a motor-cognitive functional organization of the cerebellum. Posterior cerebellar volume loss is mainly associated with cognitive impairment, whereas anterior cerebellar volume loss is associated with motor dysfunction $[6,7]$. 
The role of cerebellum in cognition and more specifically, the relationship between cerebellar abnormalities and cognitive deficits in MS remains elusive. Functional imaging and clinical studies have provided evidence for the cerebellar contribution to attention (mainly divided and alternating attention), learning and memory [8], various aspects of executive functions, such as working memory, multitasking, inhibition [9, 10], task-switching [11], verbal fluency and concept formation [8]. Language impairments (writing and speech) can also be related to cerebellum pathology [12]. In individuals with dyslexia cerebellar dysfunction has been suggested as a potential etiological factor [13-15]. Furthermore, cerebellar lesions have also been related to visuospatial deficits and impaired performance in judgment of line orientation, mental rotation and cube drawing tasks $[8,16]$. Finally, a constellation of cognitive deficits involving the cerebellum has been described by Schmahmann and Sherman [17], who introduced the cerebellar cognitive- affective syndrome (CCAS), a condition observed in adults and children, after acquired cerebellar lesions, tumors, cerebellar degeneration, Chiari malformation, cerebellar hypoplasia and agenesis [17-19]. The symptoms involve deficits in executive functions (planning, organization, set-shifting, working memory, verbal fluency, abstract thinking, mental flexibility), difficulties with spatial cognition (organization of visual short-term memory), personality changes (disinhibition, blunted affect) and language impairments (dysprosody and agrammatism).

The contribution of cerebellar pathology to cognitive difficulties of PwMS in particular, remains largely unknown. Of note, PwMS with cerebellar lesions have a different neurocognitive profile compared to those without cerebellar lesions, but its exact characteristics warrant further investigation $[7,20]$. In several studies, GM loss in the posterior cerebellar lobules and, more specifically, in vermis (lobule VI) was associated with impairment in information processing speed (Symbol Digit Modalities Test; SDMT) [7, 21-24]. In fact, lobule Crus I emerged among the other posterior cerebellar lobules, as an independent predictor of SDMT and California verbal learning test performance, a verbal learning and memory task [25]. The authors hypothesized that the cerebellum in PwMS is involved in the cognitive automation of a task, leaving the more demanding functions to higher-order associative cerebral areas, such as the prefrontal cortex [22].

Correlations of cerebellar volumes with disease severity and neurological disability, such as that assessed with the Expanded Disability Status Scale (EDSS) [26] were less consistent across studies, even though the trend was towards a negative relationship. For example, D' Ambrosio and his colleagues [7], found a correlation between higher cerebellar EDSS subsystem score and lower total cerebellar volume. Similar findings were reported by other studies as well $[27,28]$. On the other hand, Weier et al. [29] found no correlation between normalized total cerebellar volume and EDSS.
Studies investigating regional brain atrophy in PwMS, especially in relation to cognition have important theoretical and clinical implications. The potentially observed associations cannot infer causality. However, they constitute a starting point in our efforts to understand how different aspects of cognitive impairment can be explained by neuropathologyin the present investigation, atrophy of specific cerebellar lobules. On a clinical level, delineating the neuropathological basis in MS could potentially contribute to the discovery of new biomarkers of cognitive impairment and guide evidence-based neuropsychological rehabilitation interventions for treatment of MS cognitive deficits. Therefore, studying cerebellar focal atrophy could be of both theoretical and clinical importance.

Given the limited information on the cerebellar pathology expressed as cerebellar atrophy in MS and the much-disputed role of the cerebellum in cognitive functioning our aim was twofold: first, we wanted to determine the extent of cerebellar atrophy in patients with MS using a novel automatic magnetic resonance imaging (MRI) segmentation analysis, and second to investigate if cerebellar atrophy is related to cognitive deficits-measured with widely used neuropsychological procedures and experimental computerized tasks - and to the disability status of PwMS, assessed with the EDSS. We expected that PwMS will exhibit low WM and GM cerebellar volumes and cerebellar cortical atrophy and that both indexes (CGMV and CWMV), as well as cerebellar cortical thickness, will be correlated with performances on cognitive tasks. Finally, an association of EDSS with CGMV and CWMV indexes was expected.

\section{Materials and methods \\ 2.1 Participants}

A total of 24 (14 female) patients with MS and mean age of 38.83 years $(S D=9.9)$, were recruited from the outpatient clinic of the Multiple Sclerosis Center of the 2nd Department of Neurology of the AHEPA University Hospital of Thessaloniki, in Greece, between November 2019 and June 2020. All patients underwent a neurological examination, including the EDSS assessment, by a neurologist specializing in MS. Mean disease duration was 11 years $(S D=5.5)$ and median EDSS was 5 (range 1.5-7). Written informed consent was obtained from all participants prior to the assessment. The present study was approved by the local Ethical Committee (4.291/4) and was performed in accordance with the Declaration of Helsinki. A complete list of the demographic and clinical information of all patients with MS included in the study is summarized in Table 1.

Patients were diagnosed with MS based on the revised McDonald criteria [30]. Inclusion criteria were a recent MRI (within 3 months), and no clinical relapses and steroid treatment within the preceding 1 month. Patients with a history of psychiatric disorder, epilepsy, substance abuse and alcoholism and visual impairment that may interfere with cognitive testing were excluded from the study. 


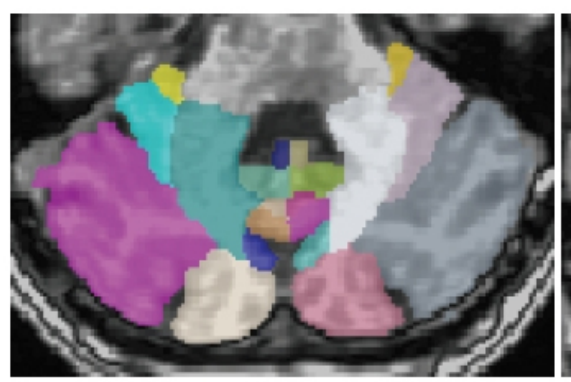

A

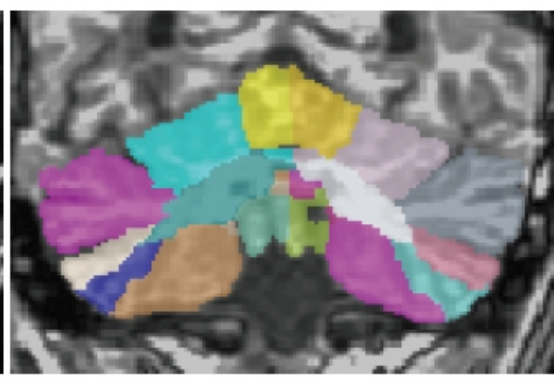

B

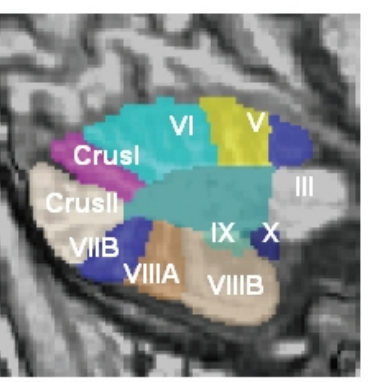

Tissue classification
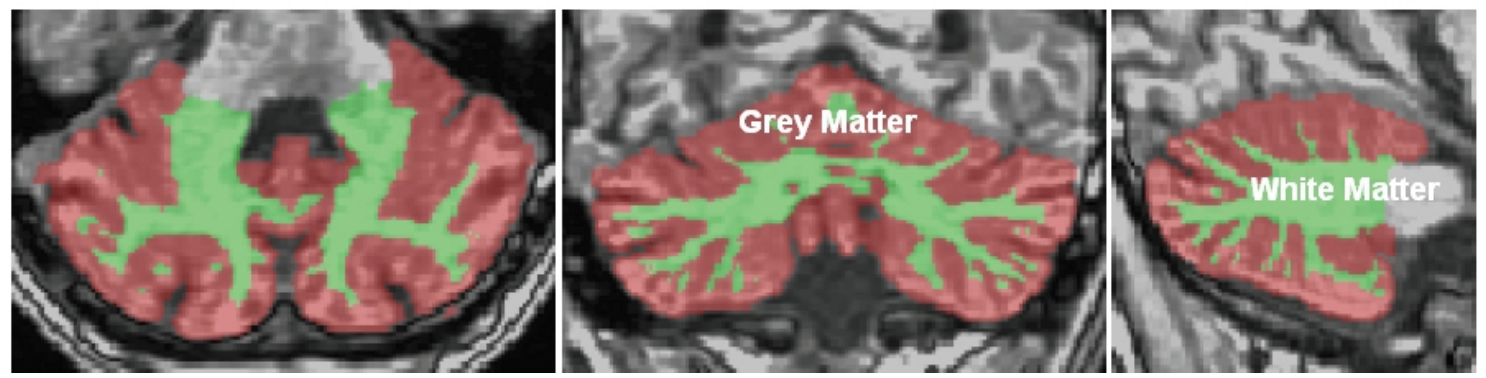

Fig. 1. Cerebellar magnetic resonance imaging (MRI) segmentation map of a representative MS patient provided by the brain volumetric program CERES. The different colors show the lobular parcellation. (A) axial, (B) coronal and (C) sagittal.

\subsection{Disease progression and disability}

The Expanded Disability Status Scale (EDSS) was used to assess disease progression and disability via seven functional domains-visual (optic), brainstem (cranial nerve function including speech and swallowing), pyramidal (motor function), cerebellar (coordination), sensory (touch and pain), bowel and bladder, and cerebral (cognition and mood). Each domain is scored from 0 (no disability) to 5 or 6 (severe disability). The total EDSS score ranges from 0 (normal examination) to 10 (death) [26].

\subsection{Neuropsychological assessment}

A list of the neuropsychological tasks administered in each cognitive domain is presented in Table 2. The neuropsychological assessment included the Brief International Cognitive Assessment for MS (BICAMS) and Brain Health Assessment (BHA-TabCAT).

The BICAMS $[31,32]$ is a paper and pencil battery, validated in the Greek population [33] that includes three tests: SDMT, the California Verbal Learning Test-2 (CVLT-2) validated in the Greek language [34]-hereinafter referred to as GVLT (total score across the five learning trials, immediate recall)- and the Brief Visuospatial Memory Test-Revised (BVMT-R) (total score across the three trials) [35].

The BHA [36] is a 10-minute tablet-administered cognitive assessment developed at UCSF and programmed in the TabCAT software platform. It consists of five subtests: Favorites (associative memory), Match (executive function and speed), Line Orientation (visuospatial), and Animal Fluency (language). For the purposes of the present study, we added an additional executive function/sustained attention test, the Set-Shifting task, also on TabCAT. We used the Greek translation of the platform (see the Supplementary material for the translation procedure). In more detail, from the Favorites task, the two variables included in the analyses were (a) the total number of correct responses across both learning trials and the delay trial and (b) recognition score which is a d-prime score/variable; from the Match test, the variable used in the analyses was the total correct score in two minutes; from the Line Orientation test, the variable used was the threshold score, which represents for the line orientation the average angle difference between the non-match orange line and the white line at which probability of the examinee's correct response is $75 \%$. Finally, for the Set-Shifting task, the variable used was the set shifting score that combines accuracy and reaction time metrics.

We added to the neuropsychological assessment the Letter Fluency task, to complement the semantic condition of the verbal fluency test. The version administered was developed by Kosmidis et al. [34], where the patient is requested to orally produce words that begin with the Greek letter " $\mathrm{X}$ " in one minute.

Participants also completed the Cognitive Reserve Index Questionnaire (CRI-q), Greek adaptation, which is administered through a semi-structured interview [37]. CRI-q assesses three domains as indexes of cognitive reserve: education, working activity and leisure time activities. 
Table 1. Demographic and clinical characteristics of the whole cohort of MS patients $(\mathrm{N}=24)$.

\begin{tabular}{lcc}
\hline & Mean (SD) or frequency (\%) & Range \\
\hline Age (years) & $38.83(9.9)$ & $21-57$ \\
Education level in years & $13.25(2.3)$ & $9-18$ \\
Gender (male/female) & $10 / 14(41.7 \% / 58.3 \%)$ & \\
Hand (right/left) & $21 / 3(87.5 \% / 12.5 \%)$ & \\
MS type (RRMS/SPMS/PPMS) & $13 / 2 / 9(54.2 \% / 8.3 \% / 37.5 \%)$ & \\
EDSS & $4.8(1.7)$ & $1.5-7$ \\
Disease duration (years) & $11(5.5)$ & $2-23$ \\
MFIS total & $37.8(19.4)$ & $36-100$ \\
CRIq & $95.3(7)$ & $84-107$ \\
DASS-21 & $17.8(2.8)$ & $0-50$ \\
BDI & $4.6(4.1)$ & $0-14$ \\
DMT (yes/no) & $19 / 5(79.2 \% / 20.8 \%)$ & \\
Natalizumab & $8(39.3 \%)$ & \\
Ocrelizumab & $7(29.2 \%)$ & \\
Fingolimod & $1(4.2 \%)$ & \\
Alemtuzumab & $1(4.2 \%)$ & \\
Interferon Beta-1a & $1(4.2 \%)$ & \\
Glatiramer acetate & $1(4.2 \%)$ & \\
\hline
\end{tabular}

Note: Data are shown as M, mean; SD, Standard Deviation or as frequency in \%; EDSS, Expanded disability status scale; MFIS, Modified Fatigue Impact Scale total; CRIq, Cognitive Reserve Index questionnaire; DASS-21, Depression, Anxiety, Stress Scale; BDI, Beck Depression Inventory; DMT, disease modifying therapy.

Finally, we included in the study three self-assessment questionnaires (Greek adaptations). The Modified Fatigue Impact Scale (MFIS) was administered for the assessment of fatigue [38] and the Depression, Anxiety, Stress Scale (DASS21) [39] and the Beck Depression Inventory (BDI) for the assessment of the mood [40].

2.4 Magnetic resonance imaging acquisition and volumetric analysis

All MRI scans were performed in a 1.5 Tesla Philips MRI scanner, as part of the routine follow up of each patient. No scan presented new or enlarging $\mathrm{T} 2$ lesions or gadolinium enhancing T1 lesions. All scans were performed with the same imaging protocol. After checking for movement artifacts, we used 3 dimensional T1-weighted sagittal brain images (160 slices, with a slice thickness of $1.2 \mathrm{~mm}$ and $1.2 \mathrm{~mm}$ spacing between slices). Volumetric analysis of the cerebellum was performed using the CERES [41] pipeline of the VolBrain automated brain volumetry system [42] (see also Fig. 1). In brief, pseudo- anonymized images were automatically segmented according to high resolution cerebellum atlases [43]. The total cerebellar volume, cortical thickness and lobule volumes were calculated as absolute $\left(\mathrm{cm}^{3}\right)$ and relative to total intracranial volume (\%) values.

\subsection{Statistical analyses}

Statistical analyses were performed with the Statistical Package for Social Sciences (SPSS), version 25.0 for Windows (IBM Corp., Armonk, NY, USA). Normal distribution was tested for all variables with the Shapiro-Wilk test. Demo- graphic and clinical characteristics of the patients were presented as means (SD) or frequencies (\%). The proportion of patients whose cerebellar volumes and cortical thickness were below the expected bounds based on their age and sex, were automatically provided by the CERES tool (VolBrain). The association of CWMV, CGMV and cerebellar cortical thickness with clinical and neuropsychological measures was estimated with Pearson's correlation coefficient (r). When CGMV, CWMV and cerebellar cortical thickness were associated with clinical and neuropsychological measures, a linear regression model including age, sex and years of education was run to control for the potential effects of demographic variables on the observed associations. Statistical significance was set at $p<0.05$.

\section{Results \\ 3.1 Neuropsychological performances}

Mean performances and standard deviations on neuropsychological tests are presented in Table 3.

\subsection{Volumetric measures}

Cerebellar volumes for each lobule obtained are presented (Fig. 1) in absolute value (measured in $\mathrm{cm}^{3}$ ) and in relative value (measured in relation to the total intracranial volume). Cortical thickness was normalized in relation to the cube root of the intracranial volume (relative value). Mean cerebellar WM volume (CWMV) and GM volume (CGMV) in $\mathrm{cm}^{3}$ was $125.4(S D=20.6)$ and $90.7(S D=17.1)$, whereas the relative values were $9.4(S D=1.04)$ and $6.8(S D=0.99)$ respectively. Mean normalized cerebellar cortical thickness was 2.4 $\mathrm{mm}(S D=1.2)$. No significant differences in either CGMV or CWMV were observed between patients with relapsingremitting Multiple Sclerosis (RRMS), secondary progressive Multiple Sclerosis (SPMS) and primary- progressive Multiple Sclerosis (PPMS). Mean total intracranial volume (in $\mathrm{cm}^{3}$ ) was $1333.8(S D=140.6)$. Mean global and regional cerebellar volumes $\left(\right.$ in $\mathrm{cm}^{3}$ ) appear in Table 4 .

In lobule $\mathrm{X}$, all patients showed reduced GM volumes and $96 \%$ of them showed reduced WM volumes (total, left, right) (see Table 5).

Cerebellar subregions with cortical thickness below the bounds expected for age and sex are summarized in Table 6. Diffuse regional cortical thinning has been observed in all lobules apart from lobule I-II for over $75 \%$ of the patients with most of them showing cortical thinning in lobule X.

\subsection{Correlations}

Since most of PwMS showed reduced WMV/GMV and lower cortical thickness in lobules I-II, III, VI, VIII A and $\mathrm{X}$, we focused our analysis on these subregions to further examine potential correlations with neuropsychological performances. In order to study the relationship between WMV/GMV and cortical thickness with the clinical and neuropsychological measures, a series of Pearson's $r$ correlation coefficients were calculated followed by linear regression analysis to control for demographic factors such as age, sex, 
Table 2. List of neuropsychological tests administered.

\begin{tabular}{|c|c|c|}
\hline \multirow{2}{*}{ Domain } & Paper and pencil & Computerized \\
\hline & BICAMS & BHA \\
\hline Learning and memory & $\begin{array}{l}\text { - } \quad \text { Brief Visuospatial Memory Test } \\
\text { - } \quad \text { Greek Verbal Learning test }\end{array}$ & - $\quad$ Favorites \\
\hline Visuospatial abilities & - Brief Visuospatial Memory Test & Line Orientation \\
\hline Language & - $\quad$ Phonemic Fluency & - $\quad$ Animal Fluency \\
\hline Processing speed/executive functions & - $\quad$ Symbol Digit Modalities Test & $\begin{array}{ll}- & \text { Match Test } \\
\text { - } & \text { Set-Shifting }\end{array}$ \\
\hline
\end{tabular}

Table 3. Summary of neuropsychological test results of MS patients $(\mathrm{N}=24)$.

\begin{tabular}{lcc}
\hline Neuropsychological tests & Mean (SD) & Range \\
\hline SDMT & $42.13(13.8)$ & $19-70$ \\
BVMT-R & $19.8(9.7)$ & $0-34$ \\
GVLT & $51.6(14.3)$ & $23-77$ \\
Animal fluency & $18.3(7.3)$ & $6-38$ \\
Phonemic fluency & $8.4(5.4)$ & $0-22$ \\
Favorites (total) & $11.5(5.9)$ & $2-22$ \\
Favorites (recognition) & $5.8(1.9)$ & $1-8$ \\
Line orientation & $8282.61(4305)$ & $2500-18750$ \\
Match test & $39.8(13)$ & $8-60$ \\
Set-shifting & $6973.8(808.3)$ & $5435-8342$ \\
\hline
\end{tabular}

Note: Data are shown as M, mean; SD, Standard Deviation; SDMT, Symbol digit modalities test; BVMT-R, Brief Visuospatial Memory

Test-Revised; GVLT, Greek Verbal Learning Test.

education level. For all correlations only relative values were considered (measured in relation to the total intracranial volume).

\subsection{Relationship between CWMV and cognitive performance}

A negative correlation was found between WMV of lobule I-II and SDMT, BVMT-R, GVLT and Match tests, such that larger volumes were associated with worse performance. After controlling for age, sex and education level, this strong negative relationship remained for $\operatorname{SDMT}\left(R^{2}=0.651, \beta=-\right.$ $0.631, p=0.005)$ and BVMT-R only $\left(R^{2}=0.458, \beta=-0.707, p\right.$ $=0.002$ ) (see Table 7). A positive correlation between WMV of lobule III and Favorites recognition didn't remain statistically significant after controlling for demographic factors.

\subsection{Relationship between CGMV and cognitive performance}

Negative correlations were found between lobules I-II, III, VI and Crus I and neuropsychological scores. Larger volumes were again associated with worse performances. More specifically, GMV of lobule I-II was correlated with scores on SDMT, BVMT-R, GVLT and Match test; GMV of lobule VI with Line Orientation scores and Crus I with GVLT scores. After controlling for age, sex and education level, the correlations that remained significant were the ones between lobule I-II with SDMT $\left(R^{2}=0.414, \beta=-0.647, p=0.006\right)$ and BVMT-R $\left(R^{2}=0.476, \beta=-0.753, p=0.001\right)$ and between lobule VI and Line Orientation $\left(R^{2}=0.493, \beta=-0.539, p=\right.$ 0.019) (see Table 8).
In conclusion, the pattern of correlations was the same for grey and white matter volumes. White matter and grey matter atrophy of lobules I-II can predict higher performances on tests across different cognitive domains, such as information processing speed, whereas grey matter atrophy of lobule VI can predict higher performances on visuospatial tasks.

3.6 Relationship between cerebellar cortical thickness and cognitive performance

Cortical thickness was correlated mainly with performance on the Match test and Line Orientation. More specifically, after controlling for demographic factors, cortical thickness of the whole cerebellum, lobule I-II, lobule III, Crus I and lobule VIIIA, was inversely associated with scores on the Match test and positively with scores on Line Orientation (higher scores indicate worse performance); cortical thickness of lobule VI was negatively correlated with the Match as well as SDMT scores. Finally, lobule $\mathrm{X}$ was negatively associated with the Match, SDMT and Phonemic fluency test scores. The observed correlations appear in Table 9, Fig. 2 and results of the linear regression analyses appear in Table 10. On further analysis, when we additionally controlled in the linear regression for disease severity and neurological disability, assessed with EDSS, the results remained unchanged with very small variations in the significance value. For example, the $p$-value between SDMT scores and cortical thickness of lobule VI and lobule $\mathrm{X}$ was 0.022 and 0.027 respectively, instead of 0.023 and 0.020 (see Table 10). Furthermore, although the present investigation is exploratory in nature, when we applied correction for multiple comparisons, most of the observed correlations remained significant. We applied partial Bonferroni correction that accounts for both the number of the comparisons conducted (10) and the mean correlation among the outcome variables (mean Pearson $\mathrm{r}=0.39$ ), and this yielded a partial Bonferroni adjusted p-value of 0.01 (http://www.quantitativeskills.com/sisa/calc ulations/bonfer.htm) (see Tables 7,8,9).

\subsection{EDSS associations with clinical - neuropsychological tests}

As expected, after controlling for demographic variables (age, sex, years of education), EDSS was positively correlated with fatigue $\left(R^{2}=0.445, \beta=0.460, p=0.022\right)$ and negatively correlated with the SDMT $\left(R^{2}=0.438, \beta=-0.453, p=0.025\right)$, $\operatorname{BVMT}-\mathrm{R}\left(R^{2}=0.427, \beta=-0.428, p=0.031\right)$ and the Match test $\left(R^{2}=0.661, \beta=-0.498, p=0.018\right)$. Correlations with all other cognitive tests were not significant, all $p>0.05$. 
Table 4. Measurements of global and regional cerebellar volumes $\left(\mathrm{cm}^{3}\right)$ of MS patients $(\mathrm{N}=24)$. Means (SDs).

\begin{tabular}{|c|c|c|c|c|c|c|}
\hline \multirow{3}{*}{ Cerebellar structure } & \multicolumn{6}{|c|}{ Mean (SD) } \\
\hline & \multicolumn{3}{|c|}{ Grey matter } & \multicolumn{3}{|c|}{ White matter } \\
\hline & Total & Left & Right & Total & Left & Right \\
\hline Whole cerebellum & $90.7(17.1)$ & $45.7(9.1)$ & $45(8.3)$ & $125.4(20.6)$ & $62.9(10.7)$ & $62.4(10.1)$ \\
\hline Lobule I-II & $0.03(0.03)$ & $0.03(0.07)$ & $0.04(0.01)$ & $0.04(0.04)$ & $0.02(0.02)$ & $0.02(0.02)$ \\
\hline Lobule III & $1(0.2)$ & $0.5(0.1)$ & $0.5(0.1)$ & $1.3(0.3)$ & $0.6(0.2)$ & $0.8(0.3)$ \\
\hline Lobule IV & $3.36(0.56)$ & $1.65(0.32)$ & $1.71(0.28)$ & $3.9(0.59)$ & $1.9(0.33)$ & $2(0.31)$ \\
\hline Lobule V & $6.23(1.1)$ & $3.13(0.54)$ & $3.11(0.57)$ & $7.52(1.23)$ & $3.73(0.61)$ & $3.79(0.67)$ \\
\hline Lobule VI & $14.5(3.7)$ & $7.3(2)$ & $7.1(1.9)$ & $16.6(3.9)$ & $8.3(2.1)$ & $8.2(2.1)$ \\
\hline Crus I & $19.4(5.5)$ & $10.1(3.1)$ & $9.4(2.7)$ & $24(5.8)$ & $12.6(3.2)$ & $12(2.9)$ \\
\hline Crus II & $13.04(3.16)$ & $6.68(1.7)$ & $6.38(1.62)$ & $15.45(3.45)$ & $7.91(1.8)$ & $7.54(1.86)$ \\
\hline Lobule VIIB & $8.02(1.58)$ & $3.91(0.76)$ & $4.1(0.91)$ & $9(1.71)$ & $4.42(0.84)$ & $4.58(0.97)$ \\
\hline Lobule VIIIA & $10.1(2.3)$ & $5.1(1.2)$ & $5.1(1.2)$ & $11.4(2.6)$ & $5.7(1.4)$ & $5.7(1.3)$ \\
\hline Lobule VIIIB & $7.6(1.58)$ & $3.89(0.9)$ & $3.71(0.76)$ & $7.6(1.58)$ & $3.89(0.9)$ & $3.71(0.76)$ \\
\hline Lobule IX & $7.49(1.98)$ & $3.66(0.96)$ & $3.84(1.04)$ & $7.49(1.98)$ & $3.66(0.96)$ & $3.84(1.04)$ \\
\hline Lobule X & $1.6(0.4)$ & $0.8(0.3)$ & $0.8(0.2)$ & $1.6(0.5)$ & $0.8(0.3)$ & $0.8(0.2)$ \\
\hline
\end{tabular}
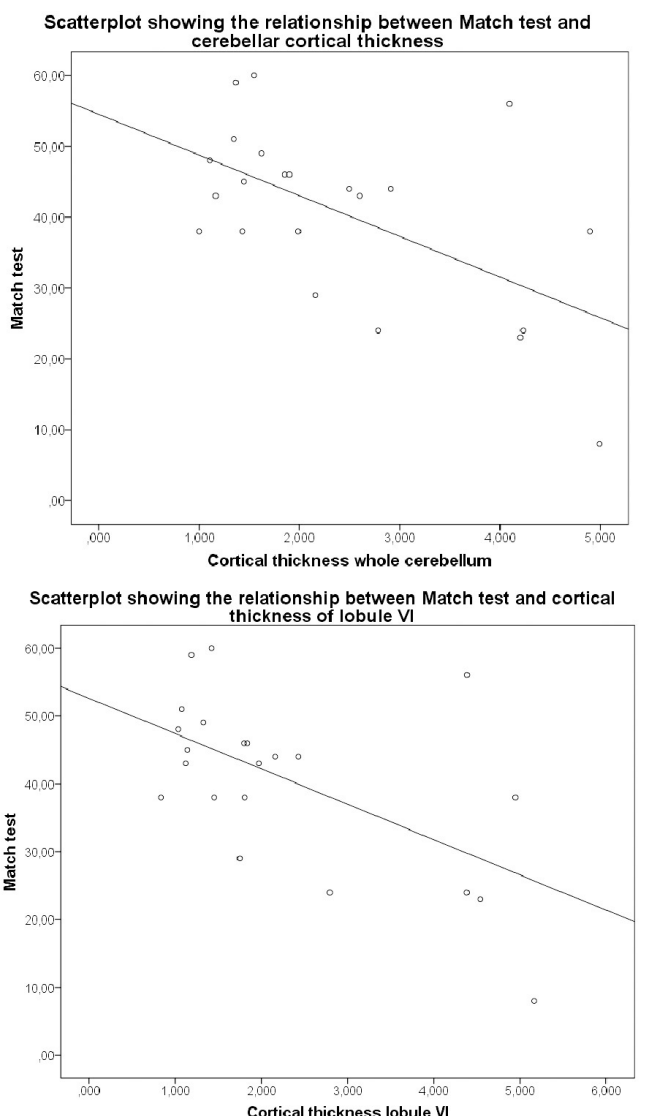

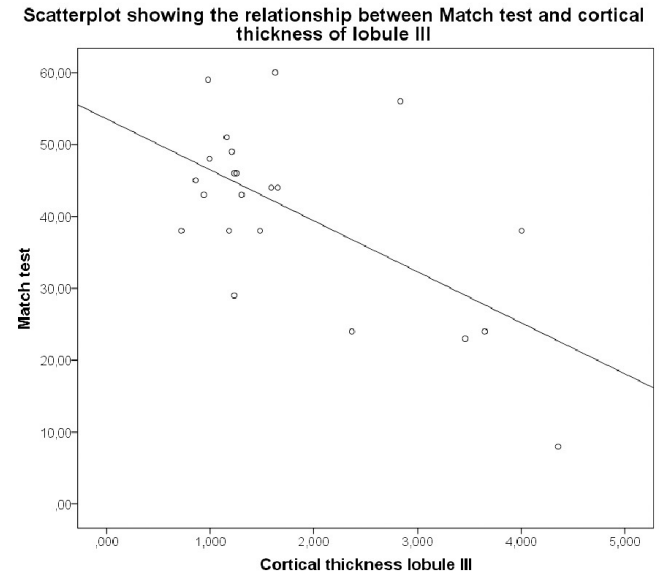

Scatterplot showing the relationship between Match test and cortical

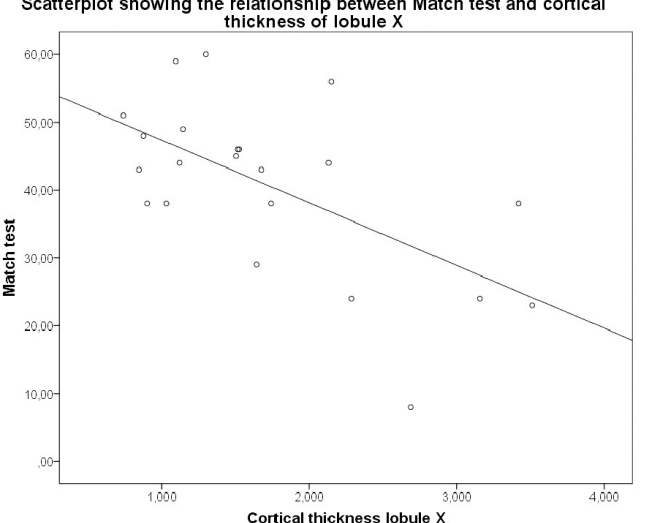

Fig. 2. Scatterplots showing the relationship (Pearson's correlation $\mathbf{r}$ ) between Match test and the whole cerebellum and lobules III, VI and X.

\subsection{EDSS associations with cerebellar volumes}

The associations of EDSS with cerebellar volumes of all cerebellar lobules (I-X) was examined with Pearson's $r$ correlational analyses. The lobule that was mainly associated with EDSS was lobule IX. Significant negative correlations were found between EDSS and right cerebellar WM and GM volume, as well as, with the total volume of lobule IX. However, after controlling for demographic variables, the observed associations failed to reach statistical significance $(p>0.05)$.

\section{Discussion}

In the present exploratory study, we performed a comprehensive volumetric assessment of the cerebellum and investigated its association with cognitive functioning and disability 
Table 5. Percentages of cerebellar white and grey matter volumes below age- and sex-adjusted bounds in MS patients $(\mathrm{N}=24)$.

\begin{tabular}{lcccccc}
\hline \multirow{2}{*}{ Cerebellar structure } & \multicolumn{3}{c}{ WMV } & \multicolumn{3}{c}{ GMV } \\
\cline { 2 - 7 } & Total & Left & Right & Total & Left & Right \\
\hline Cerebellum & $9 \%$ & $17 \%$ & $9 \%$ & $26 \%$ & $22 \%$ & $22 \%$ \\
Lobule I-II & $30 \%$ & $13 \%$ & $35 \%$ & $22 \%$ & $13 \%$ & $26 \%$ \\
Lobule III & $22 \%$ & $22 \%$ & $13 \%$ & $0 \%$ & $0 \%$ & $0 \%$ \\
Lobule IV & $0 \%$ & $9 \%$ & $0 \%$ & $4 \%$ & $4 \%$ & $0 \%$ \\
Lobule V & $0 \%$ & $0 \%$ & $0 \%$ & $0 \%$ & $0 \%$ & $0 \%$ \\
Lobule VI & $17 \%$ & $26 \%$ & $22 \%$ & $22 \%$ & $22 \%$ & $17 \%$ \\
Crus I & $9 \%$ & $9 \%$ & $17 \%$ & $30 \%$ & $17 \%$ & $22 \%$ \\
Crus II & $4 \%$ & $4 \%$ & $4 \%$ & $4 \%$ & $4 \%$ & $9 \%$ \\
Lobule VIIB & $4 \%$ & $0 \%$ & $4 \%$ & $4 \%$ & $4 \%$ & $0 \%$ \\
Lobule VIIIA & $17 \%$ & $13 \%$ & $13 \%$ & $13 \%$ & $9 \%$ & $13 \%$ \\
Lobule VIIIB & $9 \%$ & $4 \%$ & $9 \%$ & $4 \%$ & $4 \%$ & $9 \%$ \\
Lobule IX & $0 \%$ & $4 \%$ & $4 \%$ & $0 \%$ & $0 \%$ & $0 \%$ \\
Lobule X & $96 \%$ & $96 \%$ & $96 \%$ & $100 \%$ & $100 \%$ & $100 \%$ \\
\hline
\end{tabular}

Table 6. Cerebellar cortical thinning below the bounds

\begin{tabular}{lccc}
\multicolumn{4}{l}{ expected for their age and sex in } \\
\hline Cerebellar structure & Mean & Left & Right \\
\hline Cerebellum & $78 \%$ & $83 \%$ & $78 \%$ \\
Lobule I-II & $74 \%$ & $74 \%$ & $74 \%$ \\
Lobule III & $74 \%$ & $78 \%$ & $74 \%$ \\
Lobule IV & $74 \%$ & $74 \%$ & $74 \%$ \\
Lobule V & $74 \%$ & $74 \%$ & $74 \%$ \\
Lobule VI & $74 \%$ & $74 \%$ & $74 \%$ \\
Crus I & $74 \%$ & $74 \%$ & $74 \%$ \\
Crus II & $87 \%$ & $87 \%$ & $87 \%$ \\
Lobule VIIB & $87 \%$ & $87 \%$ & $83 \%$ \\
Lobule VIIIA & $83 \%$ & $83 \%$ & $78 \%$ \\
Lobule VIIIB & $83 \%$ & $83 \%$ & $78 \%$ \\
Lobule IX & $56 \%$ & $61 \%$ & $61 \%$ \\
Lobule X & $93 \%$ & $93 \%$ & $87 \%$ \\
\hline
\end{tabular}

Note: Cortical thickness is normalized in relation to

the cube root of the intracranial volume (adimen-

sional). $N=24$.

status of patients with MS. The use of advanced MRI techniques such as diffusion-weighted imaging and volumetric imaging, in the study of cerebellum, can reveal microstructural changes, assess regional and global volumes, as well as measure lesion load (for a review see Mormina et al. [44]). The semiautomatic computerized volumetric method used in this study to assess lobular GM and WM cerebellar volumes, is appropriate for the investigation of cerebellar morphometry per se, objectively and reliably quantifies cerebellar tissues and is also expected to provide important biomarkers for cerebellar disease [45].

A major finding of the present investigation is that almost all PwMS had reduced GMV and WMV in lobule X and over $75 \%$ presented with widespread cerebellar cortical thinning. This was expected, as cerebellum is among the brain structures most vulnerable to focal and diffuse (i.e., thin- ning) damage in MS compared to other brain regions [27]. Furthermore, cerebellar pathology involving neuroinflammatory processes, as well as white and grey matter demyelination is a finding well established in MS (for a review see Wilkins et al. [20]).

A second finding is that disease severity and disability, measured with the EDSS was positively associated with fatigue and inversely associated with measures of processing speed/executive functions/associative memory (SDMT and Match). More specifically, the higher the disability, the higher the fatigue and the lower the processing speed ability. Fatigue in MS refers to low motor and/or mental energy and is one of the most common symptoms of the disease. Similarly, the primary cognitive domain affected by MS is information processing speed. Recently, Eizaguirre and colleagues also found that patients with higher EDSS and greater degree of fatigue had slow processing speed [46]. Similar findings on the correlations between disability status and cognitive impairment in MS have also been reported by others [47]. It seems that motor disability, fatigue and slow processing speed in MS are intertwined, although their underlying pathophysiologic mechanisms remain elusive.

Disease severity and disability did not correlate with cerebellar CGMV and CWMV. The absence of EDSS correlations with cerebellar volumes is in line with most previous studies. Damasceno, Damasceno, and Centers [48] found that cerebellar lesion load and not grey and white matter volumes correlated with EDSS. Other studies employing similar or different volumetric analysis of the cerebellum have also failed to detect associations between CGMV/CWMV and EDSS $[25,29,49]$. The observed lack of correlation in these studies was interpreted potentially as a result of low specificity and sensitivity of the existing imaging markers for pathology. An alternative explanation was that clinical measures, such as the EDSS, may not be sensitive enough to assess the high clinical heterogeneity of the disease. On the other hand, Calabrese and colleagues [27] and D' Ambrosio and colleagues [7], did report a correlation between cortical cerebellar volume and EDSS, although a modest one. A potential explanation for the discrepant findings of the above-mentioned studies, may be the inclusion of patients with different disease severity. At the early stages of the disease, disability could be mainly determined by subcortical white matter lesions whereas at the later stages, it could be determined by the extent of widespread cortical pathology [50].

Regarding the associations between cerebellar volumetric indexes and cognition, we found that WMV and GMV of lobule I-II were both negatively correlated with SDMT and BVMT-R. This negative relationship observed between WMV and GMV of lobule I-II in the anterior cerebellum and information processing speed test (SDMT), as well as visuospatial memory (BVMT-R), means that PwMS with more white and grey matter atrophy in this lobule had higher scores on processing speed and visuospatial tasks and vice-versa; that is, larger volume correlated with worse performance on 
Table 7. Pearson's correlation coefficient $(r)$ between cerebellar global and regional white matter volumes and neuropsychological measures $(\mathrm{N}=24)$.

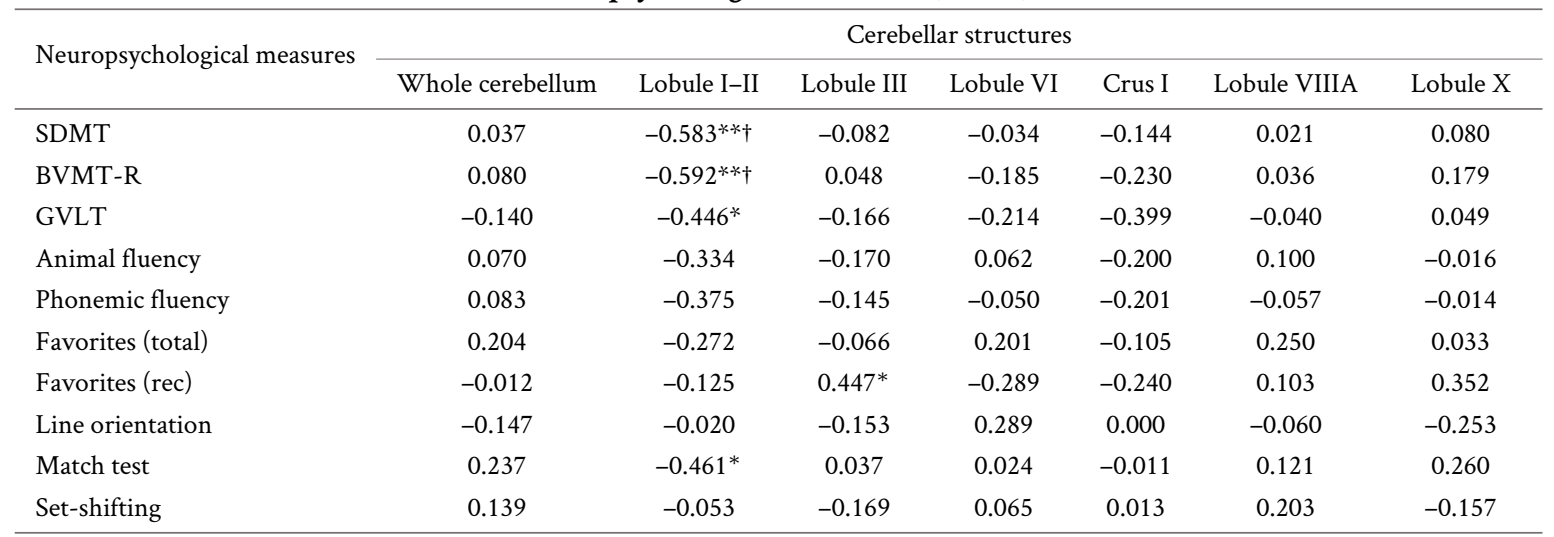

Note: *, Correlation is significant at the 0.05 level (2-tailed); ${ }^{* *}$, Correlation is significant at the 0.01 level (2-tailed). $\dagger$, Correlation is significant after correction for multiple comparisons. SDMT, Symbol digit modalities test; BVMT-R, Brief Visuospatial Memory TestRevised; GVLT, Greek Verbal Learning Test.

Table 8. Pearson's correlation coefficient $(r)$ between global and regional cerebellar grey matter volumes and neuropsychological measures $(\mathrm{N}=24)$.

\begin{tabular}{|c|c|c|c|c|c|c|c|}
\hline Neuropsychological measures & \multicolumn{7}{|c|}{ Cerebellar structures } \\
\hline SDMT & -0.065 & $-0.593^{* * \dagger}$ & 0.073 & -0.091 & -0.214 & 0.214 & 0.056 \\
\hline BVMT-R & -0.098 & $-0.419^{*}$ & 0.084 & -0.208 & -0.300 & 0.230 & 0.148 \\
\hline GVLT & -0.245 & $-0.604^{* * \dagger}$ & -0.059 & -0.245 & $-0.459^{*}$ & 0.081 & 0.032 \\
\hline Animal fluency & -0.100 & -0.303 & -0.297 & -0.009 & -0.261 & -0.082 & -0.046 \\
\hline Favorites (total) & -0.013 & -0.305 & -0.166 & 0.103 & -0.218 & 0.167 & -0.014 \\
\hline Favorites (rec) & -0.110 & -0.059 & 0.370 & -0.330 & -0.344 & 0.159 & 0.374 \\
\hline Line orientation & 0.114 & -0.021 & -0.212 & 0.344 & 0.118 & 0.011 & -0.233 \\
\hline Match test & 0.017 & $-0.500^{*}$ & 0.056 & -0.051 & -0.151 & 0.155 & 0.215 \\
\hline
\end{tabular}

Note: *, Correlation is significant at the 0.05 level (2-tailed); ${ }^{* *}$, Correlation is significant at the 0.01 level (2-tailed). $\dagger$, Correlation is significant after correction for multiple comparisons. SDMT, Symbol digit modalities test; BVMT-R, Brief Visuospatial Memory TestRevised; GVLT, Greek Verbal Learning Test.

cognitive tests. Even if these are unexpected findings, similar results in healthy adults have been previously reported, either for the cerebellum or other brain structures. Of note, Bernard et al. [51] found that larger volume in the anterior cerebellum was associated with worse performance on a processing speed test and larger volume in the left posterior cerebellum was associated with shorter spatial span. Foster et al. [52] found that larger hippocampal volume was associated with worse delayed recall performance. The authors of these studies provided a very interesting neurodevelopmental interpretation, namely that larger volume resulted from insufficient neural pruning process during development or even from altered neuronal migration, which might have affected cognition [51, 52]. Although these studies involved healthy adults, this could be the case with PwMS as well. That is, by analogy to the hippocampus, "an inadequately pruned cerebellum mediates cognitive functions less efficiently than a well pruned cerebellum" [52]. Therefore, despite the small sample size, our findings cannot be easily dismissed as being random. An alternative interpretation for this counterintuitive finding relies on the functional connectivity between cerebellum and higher-order cortical areas. Functional and not structural alterations in the cerebellum may be primarily related to cognitive impairment, and cerebro-cerebellar connectivity and not cerebellar atrophy may be a better predictor for cognitive impairment in MS, than atrophy in the cerebellum per se. Of note, functional neuroimaging findings appear to corroborate this interpretation. Cerebellar dysfunction or damage correlates with enhanced activity either within the cerebellum, or with other brain areas anatomical connected to the cerebellum. Interestingly, Rocca et al. [53] reported that structural alterations (i.e., atrophy) and accumulation of focal lesions in the cerebellum correlated with increased activation of prefrontal areas (inferior and superior frontal gyrus) in RRMS patients with better cognitive performances. In fact, despite the presence of neural damage, measured by 
Table 9. Pearson's correlation coefficient $(r)$ between global and regional cerebellar cortical thickness and neuropsychological measures $(\mathrm{N}=24)$.

\begin{tabular}{|c|c|c|c|c|c|c|c|}
\hline \multirow{2}{*}{ Neuropsychological measures } & \multicolumn{7}{|c|}{ Cerebellar structures } \\
\hline & Whole Cerebellum & Lobule I-II & Lobule III & Lobule VI & Crus I & Lobule VIIIA & Lobule X \\
\hline SDMT & $-0.435^{*}$ & -0.271 & $-0.429^{*}$ & $-0.471^{*}$ & $-0.441^{*}$ & -0.372 & $-0.538^{* * \dagger}$ \\
\hline BVMT-R & -0.343 & -0.364 & -0.360 & -0.406 & -0.322 & -0.301 & $-0.444^{*}$ \\
\hline GVLT & $-0.426^{*}$ & -0.196 & -0.374 & $-0.452^{*}$ & $-0.429^{*}$ & -0.356 & $-0.415^{*}$ \\
\hline Animal fluency & -0.338 & -0.323 & -0.319 & -0.348 & -0.372 & -0.282 & $-0.434^{*}$ \\
\hline Phonemic fluency & $-0.484^{*}$ & -0.488 & $-0.441^{*}$ & $-0.479^{*}$ & $-0.487^{*}$ & $-0.446^{*}$ & $-0.565^{* * \dagger}$ \\
\hline Favorites (total) & -0.394 & $-0.599^{*}$ & -0.409 & -0.388 & $-0.419^{*}$ & -0.324 & $-0.473^{*}$ \\
\hline Favorites (rec) & -0.234 & -0.396 & -0.265 & -0.296 & -0.206 & -0.195 & -0.247 \\
\hline Line orientation & $0.504^{*}$ & $0.546^{*}$ & $0.507^{*}$ & $0.483^{*}$ & $0.491^{*}$ & $0.492^{*}$ & 0.372 \\
\hline Match test & $-0.577^{* * \dagger}$ & $-0.741^{* * \dagger}$ & $-0.616^{* * \dagger}$ & $-0.579^{* * \dagger}$ & $-0.571^{* *}$ & $-0.531^{*}$ & $-0.611^{* * \dagger}$ \\
\hline Set-shifting & -0.158 & -0.304 & -0.105 & -0.139 & -0.191 & -0.068 & -0.260 \\
\hline
\end{tabular}

Note: *, Correlation is significant at the 0.05 level (2-tailed); ${ }^{* *}$, Correlation is significant at the 0.01 level (2-tailed). $\dagger$, Correlation is significant after correction for multiple comparisons. SDMT, Symbol digit modalities test; BVMT-R, Brief Visuospatial Memory Test-Revised; GVLT, Greek Verbal Learning Test.

T2 lesion load, an extensive over-activation in different brain areas (frontal, parietal and temporal) was observed in patients with MS whose performance was similar to healthy controls. On the contrary, PwMS with worse performance showed less activation [54]. In summary, the above findings suggest that a compensatory mechanism may be activated in MS, employing additional high-level cortical areas interconnected with the cerebellum, in order to cope with the cognitive demands of a task. Other fMRI studies have shown decreased functional connectivity and lower intensity of spontaneous fluctuations among different brain areas in PwMS, which in conjunction with the absence of differences between PwMS and healthy controls on cognitive tests, could indicate subclinical disability undetected by neuropsychological assessments $[50,55]$.

In the present study, inverse associations between cortical thinning of the whole cerebellum, lobule I-II, lobule III, lobule VI, Crus I and lobule VIIIA and information processing speed and verbal fluency (phonemic fluency) tasks (mainly timed tests, as well as those assessing visuospatial abilities, such as judgement of line orientation) were found. In fact, we found more and stronger correlations between cerebellar cortical thickness and cognitive performance than between white and grey matter volumes and cognitive performance. Longitudinal and cross-sectional studies have consistently reported widespread cortical thickness reduction in PwMS $[56,57]$. Cerebellar together with thalamic cortical atrophy has been detected in MS and patients with the clinically isolated syndrome compared to healthy controls revealing that regional cortical thinning is an early feature in MS and progresses along with the disease progression [58]. Specifically, cerebellar lobules VI, Crus I and VIIIa atrophy were independent predictors of 9-HPT, SDMT, BVMT and CVLT performances in PPMS patients confirming the contribution of these lobules to cognitive functions [25]. On the other hand, Calabreze et al. [27], found a different pattern of cortical thinning in cognitively unimpaired and impaired PwMS. The former presented with significant cortical thinning at frontotemporal areas, whereas the latter a more widespread cortical thinning, involving the whole brain. Perhaps, cognitive impairment in MS requires a diffuse generalized cortical thinning.

Several limitations of this study are noted. First, due to the small and uneven (as it pertains to MS subtypes), sample size, we did not investigate cerebellar volumetric differences among MS subtypes. It is, therefore, possible that distinct subtypes may present different patterns of pathology and associations with cognition. For example, we would expect greater cerebellar volume reduction in SPMS and PPMS patients in accordance with the theory that posits that atrophy is related to progressive disability $[3,27,59]$. Second, the majority of the patients included were males, contrary with the well-known female dominance in MS epidemiology. However, we did not observe any sex differences on either cerebellar volumes or test performances. Third, the present study is cross-sectional. Longitudinal investigations may shed light into how the accumulation of cerebellar pathology relates to clinical manifestations in MS. A further potential limitation may be resulting from the automated brain segmentation methods per se, as these methods require optimal imaging planes for accurate segmentation. Nevertheless, when compared to other cerebellar volumetric tools, CERES was ranked in the first two out of eight segmentation methods in accuracy and fidelity [41]. In addition, tiny cerebellar volumes, considered in the present analysis (but also previous studies), such as lobule I-II, may not produce as reliable measures as bigger volumes, i.e., lobule VI. A final methodological limitation is the lack of a control comparison group. However, we did rely on the normative data included in the CERES volbrain dataset library to estimate abnormal cerebellar volumes in our clinical sample. 
Table 10. Relationship between global and regional cortical thickness and neuropsychological measures. Results of the linear regression analysis $(\mathrm{N}=24)$.

\begin{tabular}{|c|c|c|c|c|c|c|}
\hline & \multicolumn{3}{|c|}{ Model } & \multicolumn{3}{|c|}{ Independent predictors } \\
\hline & $\mathrm{R}^{2}$ & $\mathrm{~F}$ & $p$-value & $\beta$ & $\mathrm{t}$-value & $p$-value \\
\hline \multicolumn{7}{|l|}{ Match test } \\
\hline Whole cerebellum & 0.659 & 8.229 & 0.001 & -0.781 & -5.195 & $<0.0001$ \\
\hline Sex & & & & 0.057 & 0.356 & 0.726 \\
\hline Age & & & & -0.580 & -3.729 & 0.002 \\
\hline Education & & & & 0.048 & 0.289 & 0.776 \\
\hline Lobule I-II & 0.623 & 4.125 & 0.031 & -0.772 & -3.937 & 0.003 \\
\hline Sex & & & & 0.177 & 0.716 & 0.490 \\
\hline Age & & & & -0.237 & -1.199 & 0.258 \\
\hline Education & & & & -0.083 & -0.333 & 0.746 \\
\hline Lobule III & 0.708 & 10.319 & $<0.0001$ & -0.811 & -5.861 & $<0.0001$ \\
\hline Sex & & & & 0.050 & 0.342 & 0.737 \\
\hline Age & & & & -0.568 & -3.977 & 0.001 \\
\hline Education & & & & 0.079 & 0.574 & 0.614 \\
\hline Crus I & 0.637 & 7.457 & 0.001 & -0.762 & -4.926 & $<0.0001$ \\
\hline Sex & & & & 0.052 & 0.318 & 0.755 \\
\hline Age & & & & -0.581 & -3.606 & 0.002 \\
\hline Education & & & & 0.000 & 0.002 & 0.998 \\
\hline Lobule VI & 0.683 & 9.158 & $<0.0001$ & -0.804 & -5.501 & $<0.0001$ \\
\hline Sex & & & & 0.047 & 0.307 & 0.763 \\
\hline Age & & & & -0.600 & -3.973 & 0.001 \\
\hline Education & & & & 0.066 & 0.413 & 0.685 \\
\hline Lobule VIIIA & 0.602 & 6.418 & 0.002 & -0.742 & -4.539 & $<0.0001$ \\
\hline Sex & & & & 0.054 & 0.312 & 0.759 \\
\hline Age & & & & -0.555 & -3.311 & 0.004 \\
\hline Education & & & & 0.102 & 0.571 & 0.576 \\
\hline Lobule X & 0.701 & 9.963 & $<0.0001$ & -0.818 & -5.753 & $<0.0001$ \\
\hline Sex & & & & 0.910 & 0.612 & 0.549 \\
\hline Age & & & & -0.625 & -4.229 & 0.001 \\
\hline Education & & & & -0.134 & -0.853 & 0.405 \\
\hline \multicolumn{7}{|l|}{ SDMT } \\
\hline Lobule VI & 0.450 & 3.682 & 0.023 & -0.638 & -3.405 & 0.003 \\
\hline Sex & & & & 0.010 & 0.055 & 0.957 \\
\hline Age & & & & -0.340 & -1.792 & 0.090 \\
\hline Education & & & & 0.303 & 1.606 & 0.126 \\
\hline Lobule X & 0.459 & 3.825 & 0.020 & -0.639 & -3.481 & 0.003 \\
\hline Sex & & & & 0.018 & 0.097 & 0.924 \\
\hline Age & & & & -0.346 & -1.833 & 0.083 \\
\hline Education & & & & 0.173 & 0.926 & 0.367 \\
\hline \multicolumn{7}{|l|}{ Phonemic fluency } \\
\hline Lobule X & 0.460 & 3.832 & 0.020 & -0.683 & -3.719 & 0.002 \\
\hline Sex & & & & -0.060 & -0.328 & 0.747 \\
\hline Age & & & & -0.383 & -2.033 & 0.057 \\
\hline Education & & & & 0.053 & 0.286 & 0.778 \\
\hline
\end{tabular}

Note: Match Test (BHA); SDMT, Symbol digit modalities test.

This is a preliminary and exploratory in nature study. Future work with larger clinical samples should examine the replicability of the current findings and possibly investigate the rate of cerebellar regional volume loss and its association with the rate of clinical and cognitive progression in PwMS using a longitudinal research design.

\section{Conclusions}

In conclusion, we observed cerebellar cortical, grey and white matter atrophy in PwMS and inverse associations with cognitive functions, mainly information processing speed. In our study, the "bigger is better" hypothesis, as it pertains to the associations of cerebellum with information processing speed in MS, was not confirmed. It should be noted, however, that the cause of cognitive impairment in MS is multifactorial and cannot be interpreted solely by the pathological findings captured by conventional MRI in the cerebellum, in particular. The complex relationship between a structure's volume and its functional efficiency warrants further investigation.

\section{Abbreviations}

MS, Multiple Sclerosis; BHA, Brain Health Assessment; CGMV, cerebellar grey matter volume; CWMV, cerebellar white matter volume; CNS, central nervous system; PwMS, persons with Multiple Sclerosis; CCAS, cerebellar cognitiveaffective syndrome; SDMT, symbol digit modalities test; MRI, magnetic resonance imaging; EDSS, Expanded Disability Status Scale; BICAMS, Brief International Cognitive Assessment for MS; CVLT-2, California Verbal Learning Test2; GVLT, Greek Verbal Learning Test; BVMT-R, Brief Visuospatial Memory Test-Revised; CRI-q, Cognitive Reserve Index Questionnaire; MFIS, Modified Fatigue Impact Scale; DASS-21, Depression, Anxiety, Stress Scale; BDI, Beck Depression Inventory; SPSS, Statistical Package for Social Sciences; RRMS, relapsing-remitting Multiple Sclerosis; SPMS, secondary progressive Multiple Sclerosis; PPMS, primary progressive Multiple Sclerosis.

\section{Author contributions}

PIliad conceived and designed the experiments; PIliad and CB performed the experiments and collected the data; PIliad and EA designed the methodology; EA reviewed and validated the methodology; SS analyzed the MRI data; SZ and KP contributed materials; PIliad wrote the original draft; EA, KP, PIoan, NG, CB, and SZ reviewed and edited the draft. All authors contributed to editorial changes in the manuscript. All authors read and approved the final manuscript.

\section{Ethics approval and consent to participate}

All the reported experiments on humans were conducted in accordance with the Declaration of Helsinki. Written informed consent was obtained from participants prior to the commencement of study procedures.

\section{Acknowledgment}

We would like to thank all the MS patients who accepted willingly to participate in this study.

\section{Funding}

This research received no external funding. 


\section{Conflict of interest}

The authors declare no conflict of interest.

\section{Supplementary material}

Supplementary material associated with this article can be found, in the online version, at https://www.imrpress.com/j ournal/JIN/21/1/10.31083/j.jin2101013.

\section{References}

[1] Macías Islas MÁ, Ciampi E. Assessment and Impact of Cognitive Impairment in Multiple Sclerosis: An Overview. Biomedicines. 2019; 7: 22 .

[2] Mitiku NS, Sandoval AEG, Kraft GH. Rehabilitation. In Samkoff LM, Goodman AD (eds.) Multiple Sclerosis and CNS Inflammatory Disorders (pp. 122-133). 1st edn. Wiley: Hoboken. 2014.

[3] Anderson V, Fisniku L, Altmann D, Thompson A, Miller D. MRI measures show significant cerebellar gray matter volume loss in multiple sclerosis and are associated with cerebellar dysfunction. Multiple Sclerosis Journal. 2009; 15: 811-817.

[4] Filippi M, Brück W, Chard D, Fazekas F, Geurts JJG, Enzinger C, et al. Association between pathological and MRI findings in multiple sclerosis. Lancet Neurology. 2019; 18: 198-210.

[5] Hamilton AM, Forkert ND, Yang R, Wu Y, Rogers JA, Yong VW, et al. Central nervous system targeted autoimmunity causes regional atrophy: a 9.4T MRI study of the EAE mouse model of Multiple Sclerosis. Scientific Reports. 2019; 9: 8488.

[6] Parmar K, Stadelmann C, Rocca MA, Langdon D, D’Angelo E, D'Souza M, et al. The role of the cerebellum in multiple sclerosis150 years after Charcot. Neuroscience \& Biobehavioral Reviews. 2018; 89: 85-98.

[7] D’Ambrosio A, Pagani E, Riccitelli GC, Colombo B, Rodegher M, Falini A, et al. Cerebellar contribution to motor and cognitive performance in multiple sclerosis: an MRI sub-regional volumetric analysis. Multiple Sclerosis Journal. 2017; 23: 1194-1203.

[8] Gottwald B, Wilde B, Mihajlovic Z, Mehdorn HM. Evidence for distinct cognitive deficits after focal cerebellar lesions. Journal of Neurology, Neurosurgery, and Psychiatry. 2004; 75: 1524-1531.

[9] Bellebaum C, Daum I. Cerebellar involvement in executive control. Cerebellum. 2007; 6: 184-192.

[10] Ravizza SM, McCormick CA, Schlerf JE, Justus T, Ivry RB, Fiez JA. Cerebellar damage produces selective deficits in verbal working memory. Brain. 2006; 129: 306-320.

[11] Stoet G, Snyder L. Task-Switching in Human and Nonhuman Primates: Understanding Rule Encoding and Control from Behavior to Single Neurons. In Bunge SA, Wallis JD (eds.) Neuroscience of Rule-Guided Behavior (pp. 227-254). 1st edn. New York, Oxford University Press. 2007.

[12] Grimaldi G, Argyropoulos GP, Boehringer A, Celnik P, Edwards MJ, Ferrucci R, et al. Non-invasive Cerebellar Stimulation-a Consensus Paper. Cerebellum. 2014; 13: 121-138.

[13] Baillieux H, Vandervliet EJM, Manto M, Parizel PM, De Deyn PP, Mariën P. Developmental dyslexia and widespread activation across the cerebellar hemispheres. Brain and Language. 2009; 108: 122-132.

[14] Brown WE, Eliez S, Menon V, Rumsey JM, White CD, Reiss AL. Preliminary evidence of widespread morphological variations of the brain in dyslexia. Neurology. 2001; 56: 781-783.

[15] Rae C, Harasty JA, Dzendrowskyj TE, Talcott JB, Simpson JM, Blamire AM, et al. Cerebellar morphology in developmental dyslexia. Neuropsychologia. 2002; 40: 1285-1292.

[16] Molinari M, Leggio MG. Cerebellar information processing and visuospatial functions. Cerebellum. 2007; 6: 214-220.

[17] Schmahmann JD, Sherman JC. The cerebellar cognitive affective syndrome. Brain. 1998; 121: 561-579.

[18] Levisohn L, Cronin-Golomb A, Schmahmann JD. Neuropsychological consequences of cerebellar tumour resection in children: cerebellar cognitive affective syndrome in a paediatric population. Brain. 2000; 123: 1041-1050.

[19] Schmahmann JD, Gardner R, MacMore J, Vangel MG. Development of a brief ataxia rating scale (BARS) based on a modified form of the ICARS. Movement Disorders. 2009; 24: 1820-1828.

[20] Wilkins A. Cerebellar Dysfunction in Multiple Sclerosis. Frontiers in Neurology. 2017; 8: 312.

[21] Amato MP, Bartolozzi ML, Zipoli V, Portaccio E, Mortilla M, Guidi L, et al. Neocortical volume decrease in relapsing-remitting MS patients with mild cognitive impairment. Neurology. 2004; 63: 89-93.

[22] Moroso A, Ruet A, Lamargue-Hamel D, Munsch F, Deloire M, Coupé $P$, et al. Posterior lobules of the cerebellum and information processing speed at various stages of multiple sclerosis. Journal of Neurology, Neurosurgery \& Psychiatry. 2017; 88: 146-151.

[23] Sarica A, Cerasa A, Quattrone A. The neurocognitive profile of the cerebellum in multiple sclerosis. International Journal of Molecular Sciences. 2015; 16: 12185-12198.

[24] Weier K, Penner IK, Magon S, Amann M, Naegelin Y, Andelova $\mathrm{M}$, et al.Cerebellar abnormalities contribute to disability including cognitive impairment in multiple sclerosis. PLoS ONE. 2014; 9: e86916.

[25] Cocozza S, Petracca M, Mormina E, Buyukturkoglu K, Podranski $\mathrm{K}$, Heinig MM, et al. Cerebellar lobule atrophy and disability in progressive MS. Journal of Neurology, Neurosurgery, and Psychiatry. 2017; 88: 1065-1072.

[26] Kurtzke JF. Rating neurologic impairment in multiple sclerosis: an expanded disability status scale (EDSS). Neurology. 1983; 33: 1444-1452.

[27] Calabrese M, Rinaldi F, Grossi P, Gallo P. Cortical pathology and cognitive impairment in multiple sclerosis. Expert Review of Neurotherapeutics. 2011; 11: 425-432.

[28] Fujimori J, Fujihara K, Ogawa R, Baba T, Wattjes M, Nakashima I. Patterns of regional brain volume loss in multiple sclerosis: a cluster analysis. Journal of Neurology. 2020; 267: 395-405.

[29] Weier K, Beck A, Magon S, Amann M, Naegelin Y, Penner IK, et al. Evaluation of a new approach for semi-automatic segmentation of the cerebellum in patients with multiple sclerosis. Journal of Neurology. 2012; 259: 2673-2680.

[30] Thompson AJ, Banwell BL, Barkhof F, Carroll WM, Coetzee T, Comi G, et al. Diagnosis of multiple sclerosis: 2017 revisions of the McDonald criteria. Lancet. Neurology. 2018; 17: 162-173.

[31] Benedict RHB, Amato MP, Boringa J, Brochet B, Foley F, Fredrikson $\mathrm{S}$, et al. Brief International Cognitive Assessment for MS (BICAMS): international standards for validation. BMC Neurology. 2012; 12: 55 .

[32] Langdon DW, Amato MP, Boringa J, Brochet B, Foley F, Fredrikson $\mathrm{S}$, et al. Recommendations for a Brief International Cognitive Assessment for Multiple Sclerosis (BICAMS). Multiple Sclerosis. 2012; 18: 891-898.

[33] Polychroniadou E, Bakirtzis C, Langdon D, Lagoudaki R, Kesidou $\mathrm{E}$, Theotokis $\mathrm{P}$, et al. Validation of the Brief International Cognitive Assessment for Multiple Sclerosis (BICAMS) in Greek population with multiple sclerosis. Multiple Sclerosis and Related Disorders. 2016; 9: 68-72.

[34] Vlahou CH, Kosmidis MH, Dardagani A, Tsotsi S, Giannakou M, Giazkoulidou A, et al. Development of the Greek Verbal Learning Test: reliability, construct validity, and normative standards. Archives of Clinical Neuropsychology. 2013; 28: 52-64.

[35] Benedict RHB, Schretlen D, Groninger L, Dobraski M, Shpritz B. Revision of the Brief Visuospatial Memory Test: Studies of normal performance, reliability, and validity. Psychological Assessment. 1996; 8: 145-153.

[36] Possin KL, Moskowitz T, Erlhoff SJ, Rogers KM, Johnson ET, Steele NZR, et al. The Brain Health Assessment for Detecting and Diagnosing Neurocognitive Disorders. Journal of the American Geriatrics Society. 2018; 66: 150-156.

[37] Maiovis P, Ioannidis P, Nucci M, Gotzamani-Psarrakou A, Karacostas D. Adaptation of the Cognitive Reserve Index Question- 
naire (CRIq) for the Greek population. Neurological Sciences. 2016; 37: 633-636.

[38] Bakalidou D, Voumvourakis K, Tsourti Z, Papageorgiou E, Poulios A, Giannopoulos S. Validity and reliability of the Greek version of the Modified Fatigue Impact Scale in multiple sclerosis patients. International Journal of Rehabilitation Research. 2014; 37: 271-276.

[39] Pezirkianidis C, Karakasidou E, Lakioti A, Stalikas A, Galanakis M. Psychometric Properties of the Depression, Anxiety, Stress Scales21 (DASS-21) in a Greek Sample. Psychology. 2018; 09: 2933 2950.

[40] Giannakou M, Roussi P, Kosmides ME, Kiosseoglou G, Adamopoulou A, Garyfallos G. Adaptation of the Beck Depression Inventory-II to Greek population. Hellenic Journal of Psychology. 2013; 10: 120-146.

[41] Carass A, Cuzzocreo JL, Han S, Hernandez-Castillo CR, Rasser PE, Ganz M, et al. Comparing fully automated state-of-the-art cerebellum parcellation from magnetic resonance images. NeuroImage. 2018; 183: 150-172.

[42] Manjón JV, Coupé P. volBrain: An Online MRI Brain Volumetry System. Frontiers in Neuroinformatics. 2016; 10: 30.

[43] Park MTM, Pipitone J, Baer LH, Winterburn JL, Shah Y, Chavez $\mathrm{S}$, et al. Derivation of high-resolution MRI atlases of the human cerebellum at $3 \mathrm{~T}$ and segmentation using multiple automatically generated templates. NeuroImage. 2014; 95: 217-231.

[44] Mormina E, Petracca M, Bommarito G, Piaggio N, Cocozza S, Inglese M. Cerebellum and neurodegenerative diseases: beyond conventional magnetic resonance imaging. World Journal of Radiology. 2017; 9: 371-388.

[45] Sörös P, Wölk L, Bantel C, Bräuer A, Klawonn F, Witt K. Replicability, Repeatability, and Long-term Reproducibility of Cerebellar Morphometry. Cerebellum. 2021; 20: 439-453.

[46] Eizaguirre MB, Vanotti S, Merino Á, Yastremiz C, Silva B, Alonso $\mathrm{R}$, et al. The Role of Information Processing Speed in Clinical and Social Support Variables of Patients with Multiple Sclerosis. Journal of Clinical Neurology. 2018; 14: 472-477.

[47] Andreasen AK, Spliid PE, Andersen H, Jakobsen J. Fatigue and processing speed are related in multiple sclerosis. European Journal of Neurology. 2010; 17: 212-218.

[48] Damasceno A, Damasceno BP, Cendes F. The clinical impact of cerebellar grey matter pathology in multiple sclerosis. PLoS ONE.
2014; 9: e96193.

[49] Boonstra FM, Noffs G, Perera T, Jokubaitis VG, Vogel AP, Moffat BA, et al. Functional neuroplasticity in response to cerebellothalamic injury underpins the clinical presentation of tremor in multiple sclerosis. Multiple Sclerosis Journal. 2020; 26: 696-705.

[50] Plata-Bello J, Pérez-Martín Y, Castañón-Pérez A, Modroño C Hernández-Martín E, González-Platas M, et al. The relationship between amplitude of low frequency fluctuations and gray matter volume of the mirror neuron system: Differences between low disability multiple sclerosis patients and healthy controls. IBRO Reports. 2018; 5: 60-66.

[51] Bernard JA, Leopold DR, Calhoun VD, Mittal VA. Regional cerebellar volume and cognitive function from adolescence to late middle age. Human Brain Mapping. 2015; 36: 1102-1120.

[52] Foster JK, Meikle A, Goodson G, Mayes AR, Howard M, Sünram $\mathrm{SI}$, et al. The hippocampus and delayed recall: bigger is not necessarily better? Memory. 1999; 7: 715-732.

[53] Rocca MA, Valsasina P, Martinelli V, Misci P, Falini A, Comi $\mathrm{G}$, et al. Large-scale neuronal network dysfunction in relapsingremitting multiple sclerosis. Neurology. 2012; 79: 1449-1457.

[54] Mainero C, Caramia F, Pozzilli C, Pisani A, Pestalozza I, Borriello $\mathrm{G}$, et al. FMRI evidence of brain reorganization during attention and memory tasks in multiple sclerosis. NeuroImage. 2004; 21 : 858-867.

[55] Plata-Bello J, Pérez-Martín Y, Castañón-Pérez A, Modroño C, Fariña H, Hernández-Martín E, et al. The Mirror Neuron System in Relapsing Remitting Multiple Sclerosis Patients with Low Disability. Brain Topography. 2017; 30: 548-559.

[56] Narayana PA, Govindarajan KA, Goel P, Datta S, Lincoln JA, Cofield SS, et al. Regional cortical thickness in relapsing remitting multiple sclerosis: a multi-center study. NeuroImage: Clinical. 2012 ; 2 : 120-131.

[57] Tsagkas C, Chakravarty MM, Gaetano L, Naegelin Y, Amann M, Parmar K, et al. Longitudinal patterns of cortical thinning in multiple sclerosis. Human Brain Mapping. 2020; 41: 2198-2215.

[58] Ramasamy DP, Benedict RHB, Cox JL, Fritz D, Abdelrahman N, Hussein $\mathrm{S}$, et al. Extent of cerebellum, subcortical and cortical atrophy in patients with MS: a case-control study. Journal of the Neurological Sciences. 2009; 282: 47-54.

[59] Inglese M, Petracca M, Mormina E, Achiron A, Straus-Farber R, Miron $S$, et al. Cerebellar volume as imaging outcome in progressive multiple sclerosis. PLoS ONE. 2017; 12: e0176519. 